\title{
Sleep related hypoxaemia in hypertensive and normotensive men
}

Iain C Gleadhill, Evelyn E McCrum, Chris C Patterson, G Dennis Johnston, Alun Evans, Joseph MacMahon

\begin{abstract}
Background-An association between hypertension and obstructive sleep apnoea (OSA) has been found by some researchers but remains controversial. Since such an association would have important implications for the investigation and management of hypertension, the rate of nocturnal hypoxaemic episodes has been compared in hypertensive and normotensive men.

Methods-The study was carried out in the community in Belfast and its environs. Thirty four men with mild to moderate hypertension aged 40-64 years were identified from general practice and a hypertension clinic. Normotensive men, matched for age and body mass index, were selected from a community survey. Subjects answered a sleep questionnaire and underwent overnight pulse oximetry at home. Computer analysis of the results gave the number and magnitude of dips in oxygen saturation $\left(\mathrm{SaO}_{2}\right.$ dips, $4 \%$ or greater).

Results-The median number of $\mathrm{SaO}_{2}$ dips/hour for hypertensives was $2 \cdot 0$, and for normotensives was $0 \cdot 8$. Lowest $\mathrm{SaO}_{2}$ and mean $\mathrm{SaO}_{2}$ levels were significantly lower in the hypertensive group. Only one subject had a rate of $\mathrm{SaO}_{2}$ dips/hour greater than five and symptoms suggestive of OSA. Conclusions-Both hypertensive and normotensive men had relatively few episodes of nocturnal hypoxaemia. The small increase in the rate of $\mathrm{SaO}_{2}$ dips in hypertensive subjects has not yet been fully explained. These results imply that OSA is not common in hypertensive subjects and is unlikely to be an important cause of hypertension.
\end{abstract}

Respiratory Medicine Unit, Belfast City Hospital, Belfast BT9 7AB

I C Gleadhill

J MacMahon

Department of

Therapeutics and

Pharmacology

G D Johnston

Department of

Epidemiology and

Public Health

E E McCrum

C C Patterson

A Evans

The Queen's

University of Belfast, Belfast

Reprint requests to:

Dr I C Gleadhill

Received 30 March 1992

Returned to authors

3 June 1992

Revised version received

3 December 1992

Accepted 9 December 1992

(Thorax 1993;48:534-536)

In obstructive sleep apnoea (OSA), repetitive obstruction of the upper airway results in recurrent hypoxaemic episodes and transient haemodynamic changes, including increases in pulmonary and systemic arterial pressure. ${ }^{1}$ It has been proposed that patients with this disorder, if not already hypertensive, will eventually develop sustained daytime systemic hypertension. ${ }^{2}$ In the mid 1980 s several studies of hypertensive subjects showed that up to $30 \%$ had OSA, supporting the view that this is an unrecognised cause of hypertension. $^{2-5}$ In contrast, a more recent study has shown no difference in the rate of nocturnal hypoxaemic episodes between hypertensive and normotensive subjects. ${ }^{6}$ In view of the conflicting evidence, and since an association between OSA and hypertension would have important implications for the investigation of hypertension, we compared hypertensive and matched normotensive subjects for evidence of increased nocturnal hypoxaemic episodes.

\section{Methods}

The study was carried out in Belfast and its environs between 1988 and 1990, with ethical committee approval. Men aged 40-64 years with essential hypertension were identified from several general practices or the hypertension clinic at Belfast City Hospital. Inclusion criteria were diastolic blood pressure of $90-110 \mathrm{~mm} \mathrm{Hg}$ at the end of a 4-8 week placebo run in period, then controlled by a single drug. Those with a history of secondary hypertension, significant cardiac, renal or pulmonary disease, alcohol abuse, or treatment with $\beta$ blocking drugs were excluded. Thirty four men with mild to moderate hypertension on monotherapy (thiazide diuretic in 25 , calcium antagonist in two, and ACE inhibitor in seven) entered the study.

For each hypertensive subject a randomly chosen control subject, matched for age $( \pm 2$ years) and body mass index $\left( \pm 2 \mathrm{~kg} / \mathrm{m}^{2}\right)$, was identified from a separate community survey. Exclusion criteria were identical except that those with raised blood pressure (systolic $\geqslant 140 \mathrm{~mm} \mathrm{Hg}$ and diastolic $\geqslant 90 \mathrm{~mm} \mathrm{Hg}$; or systolic $\geqslant 160 \mathrm{~mm} \mathrm{Hg}$; or diastolic $\geqslant 95 \mathrm{~mm}$ $\mathrm{Hg}$ ), or taking antihypertensive medication, were also excluded.

STUDY DESIGN

Subjects were visited twice at home: firstly, a sleep questionnaire was administered with the spouse present where possible, and measurements were made of blood pressure (single reading by Hawksley random zero sphygmomanometer), height, weight, neck circumference (by tape measure below the thyroid cartilage); secondly, a pulse oximeter (Ohmeda Biox 3700e) was set up in the sub- 
Table 1 Mean (SD) anthropometric and blood pressure results in 34 hypertensive and 34 normotensive men

\begin{tabular}{lccc}
\hline & $\begin{array}{l}\text { Hypertensive } \\
(n=34)\end{array}$ & $\begin{array}{l}\text { Normotensive } \\
(n=34)\end{array}$ & $p$ \\
\hline Age (y) & $52.9(6.3)$ & $53.0(6.5)$ & 0.65 \\
Body mass index $\left(\mathrm{kg} / \mathrm{m}^{2}\right)$ & $26.5(2.9)$ & $26.1(2.4)$ & 0.05 \\
Neck circumference (inches) & $15.8(0.6)$ & $15.9(0.7)^{\star}$ & 0.21 \\
Systolic blood pressure (mm Hg) & $141.3(19.4)$ & $132.8(11.6)$ & 0.06 \\
Diastolic blood pressure (mm Hg) & $92.4(10.2)$ & $80.4(6.6)$ & $<0.001$ \\
\hline
\end{tabular}

^Data missing on two subjects

ject's bedroom, and he was instructed to wear the finger probe overnight. Oximetry was repeated if the duration was less than five hours or if the subject slept poorly. The oximeter retains up to eight hours of data by storing the lowest oxygen saturation $\left(\mathrm{SaO}_{2}\right)$ for each 12 second period. The data were off loaded to a BBC B computer, a software program was used to identify dips in $\mathrm{SaO}_{2}$ of $4 \%$ or more, ${ }^{7}$ and the total number of $\mathrm{SaO}_{2}$ dips, duration of oximetry, and mean $\mathrm{SaO}_{2}$ were derived.

With the questionnaire, subjects were classified as having (1) habitual snoring if they reported snoring nightly or on most nights, and (2) excessive daytime sleepiness if they reported falling asleep at least once a day, or if they sometimes fell asleep involuntarily.

\section{STATISTICAL ANALYSIS}

Means for continuous variables were compared with the paired $t$ test and medians with the Wilcoxon signed rank test. Analysis of covariance was used to adjust the comparison of $\mathrm{SaO}_{2}$ dips between groups for the potentially confounding effect of body mass index; a logarithmic transformation was applied to the $\mathrm{SaO}_{2}$ dips because of skew in the distribution. McNemar's test was used to compare proportions. The relationships between the rate of $\mathrm{SaO}_{2}$ dips (on a logarithmic scale) and other variables were investigated by multiple regression analysis.

\section{Results}

Anthropometric data are shown in table 1. Hypertensive subjects had a slightly higher mean body mass index than normotensive men, but neck circumferences were the same. Diastolic blood pressure, despite treatment, was signficantly higher in the hypertensive group. Over half of each group regularly drank alcohol, and just over a quarter smoked

Table 2 Mean (SD) results of pulse oximetry for hypertensive and normotensive subjects

\begin{tabular}{lclll}
\hline & $\begin{array}{l}\text { Hypertensive } \\
(n=34)\end{array}$ & $\begin{array}{l}\text { Normotensive } \\
(n=34)\end{array}$ & $\begin{array}{l}\text { Difference (95\% } \\
\text { confidence intervals) }\end{array}$ & $p$ \\
\hline $\begin{array}{l}\text { Duration of } \\
\text { oximetry (h) }\end{array}$ & $6.06(0.81)$ & $6.12(0.88)$ & $\begin{array}{c}-0.06 \\
(-0.41,0.30)\end{array}$ & 0.74 \\
$\begin{array}{l}\text { Baseline } \mathrm{SaO}_{2}(\%) \\
\mathrm{Mean} \mathrm{SaO}_{2}(\%)\end{array}$ & $94.2(1.6)$ & $95.1(1.3)$ & $\begin{array}{c}-0.9 \\
(-1.6,-0.2)\end{array}$ & 0.02 \\
& $93.6(1.5)$ & $94.5(1.0)$ & $\begin{array}{c}-0.9 \\
(-1.5,-0.3)\end{array}$ & 0.005 \\
Lowest $\mathrm{SaO}_{2}(\%)$ & $85.0(5.6)$ & $87.8(3.9)$ & $\begin{array}{c}-2.8 \\
(-5.3,-0.3)\end{array}$ & 0.05 \\
$\begin{array}{c}\mathrm{No}_{\mathrm{SaO}} \text { dips/hour } \\
\text { (median quartiles) }\end{array}$ & $\begin{array}{l}2.00 \\
(1.11,3.51)\end{array}$ & $\begin{array}{c}0.83 \\
(0.48,1.45)\end{array}$ & $\begin{array}{l}1.17 \\
(0.45,2.05)\end{array}$ & 0.005 \\
\hline
\end{tabular}

$\mathrm{SaO}_{2}$-arterial oxygen saturation. cigarettes. Questionnaire responses from hypertensive and normotensive subjects showed no difference in excessive daytime sleepness $(50 \% v 41 \%$ respectively) or insomnia $(18 \% \quad v \quad 24 \%)$. Habitual snoring was reported by $50 \%$ of hypertensive and $26 \%$ of normotensive subjects $(p=0 \cdot 06)$.

The results of pulse oximetry are shown in table 2. Mean $\mathrm{SaO}_{2}$ levels were lower in hypertensive than in normotensive subjects, as were the lowest $\mathrm{SaO}_{2}$ levels. Although baseline $\mathrm{SaO}_{2}$ levels were lower in hypertensive men, baseline hypoxaemia was not observed (lowest hypertensive was $91 \%$, lowest normotensive $92 \%$ ). The median number of $\mathrm{SaO}_{2}$ dips/hour was 2.0 for hypertensive subjects and $\mathbf{0 . 8}$ for normotensive controls. Analysis of covariance indicated that, after adjustment for body mass index, the ratio of dips/hour in the hypertensive group relative to the normotensive group was 1.9 (confidence intervals $1 \cdot 2,2 \cdot 8$ ).

From a clinical standpoint only one subject had five or more $\mathrm{SaO}_{2}$ dips/hour in conjunction with symptoms suggestive of obstructive sleep apnoea (habitual snoring and excessive daytime sleepiness). This was a hypertensive subject with 8.6 $\mathrm{SaO}_{2}$ dips/hour. Three other hypertensive patients and none of the normotensive controls had five or more $\mathrm{SaO}_{2}$ dips/hour without symptoms. Multiple regression analysis showed that body mass index and neck circumference were individually predictive of the rate of $\mathrm{SaO}_{2}$ dips in the hypertensive group. When body mass index was included in the regression model, the additional predictive value obtained by adding neck circumference was not statistically significant. Each unit increase in body mass index was associated with a $13 \%$ increase in the rate of $\mathrm{SaO}_{2}$ dips.

\section{Discussion}

Both hypertensive and normotensive subjects had relatively few episodes of nocturnal hypoxaemia. Although the rate of $\mathrm{SaO}_{2}$ dips was higher in hypertensive than normotensive subjects, it was not increased to a level where it was likely to have caused elevation of blood pressure.

It has generally been thought that accurate diagnosis of sleep apnoea and other sleep disorders requires detailed monitoring of respiratory and EEG parameters (polysomnography). Pulse oximetry alone, however, identifies most episodes of apnoea and hypopnoea and diagnoses $66-75 \%$ of cases of sleep apnoea syndrome. ${ }^{89}$ Because it is portable and easy to use, the pulse oximeter has advantages for domiciliary studies of this kind.

Several earlier studies showed that almost a third of hypertensives had OSA. ${ }^{2-5}$ Using a threshold of 10 apnoeic episodes/hour of sleep, Fletcher et al reported OSA in $30 \%$ of hypertensive cases, ${ }^{3}$ whereas using a level of $10 \mathrm{SaO}_{2}$ dips/hour we found no cases. Reducing the threshold to five $\mathrm{SaO}_{2}$ dips/hour identified only four cases (12\%). Breathing 
disturbances in the study by Fletcher et al occurred at a mean rate of 10.0 /hour (18.1/hour for apnoeas plus hypopnoeas), in the study by Kales et $a l^{2}$ at a rate of $11.0 /$ hour, but in the present study the rate was $2 \cdot 6 /$ hour (median $2 \cdot 0 /$ hour). Fletcher et al used "apnoea prone" volunteers, while in the study by Kales et al subjects had more severe hypertension requiring more than one drug. Our patients were on monotherapy, usually a thiazide, and there was no apparent selection bias. Another partial explanation for the discrepancy may be that polysomnography, unlike pulse oximetry, usually requires the supine position which increases the apnoea rate. ${ }^{10}$

Our study, with similar methodology, supports that of Warley et $\mathrm{al}^{6}$ in finding - in clinical terms-a virtually normal rate of nocturnal hypoxaemic episodes in hypertensive subjects. In that study the mean rates of $\mathrm{SaO}_{2}$ dips (4\% or greater) in 30 hypertensive men and 30 normotensive men were 1.49 and $1 \cdot 72$ /hour respectively (compared with 2.6 and 1.2 /hour in our study). These rates are all low compared with the earlier studies mentioned. Our normotensive subjects had an even lower rate than the hypertensive patients; this difference may indicate a true association between hypertension and sleep related breathing disturbances. Alternatively, because of their lower baseline $\mathrm{SaO}_{2}$ hypertensive subjects may have been more vulnerable to subsequent arterial oxygen desaturation because of the shape of the oxyhaemoglobin dissociation curve. The difference in baseline $\mathrm{SaO}_{2}$ between the groups, however, was small and unlikely to have influenced the rate of $\mathrm{SaO}_{2}$ dips. Although an effect from drug therapy is possible ${ }^{11}$, we excluded patients on $\beta$ blockers and feel any effect is likely to be insignificant. Whatever the explanation, the somewhat higher rate of $\mathrm{SaO}_{2}$ dips in hypertensive patients may be of physiological interest, but 'would seem unlikely to have contributed significantly to the development of hypertension in these patients.

Habitual snoring was reported by $50 \%$ of hypertensive and $26 \%$ of normotensive subjects. Although not significantly different, this trend fits with epidemiological studies which show an association between snoring and hypertension, and lead some to suggest a causative role for snoring, perhaps as a result of OSA (present in a proportion of snorers). ${ }^{12}{ }^{13}$ It is possible that snoring, without OSA, may lead to significant haemodynamic and neuroendocrine changes. ${ }^{14} 15$ Others have argued that the association between snoring and hypertension largely reflects the presence of confounding variables such as male gender or obesity. ${ }^{16}$

In this study men with mild to moderate essential hypertension had only slightly increased rates of nocturnal hypoxaemic episodes, implying that OSA is infrequently found in these subjects. OSA would therefore seem unlikely to be an important cause of hypertension. The small difference in the rate of hypoxaemic episodes between hypertensive and normotensive subjects, and the percentages with habitual snoring, may merit further enquiry. On the basis of these results, however, sleep studies are not routinely indicated in the investigation of hypertension.

This research was supported by a grant from the Northern Ireland Chest, Heart and Stroke Association, and additional funding was provided by Allen and Hanburys Ltd. We would also like to thank Dr John Stradling for advice concerning the analysis of the oximetry recordings, and Mrs Heather Porter for preparing the manuscript.

Coccagna G, Mantovani M, Brignani F, Parchi C, Lugaresi $E$. Continuous recording of the pulmonary and systemic arterial pressure during sleep in syndromes of hypersomnia with periodic breathing. Bull Physiopathol 1972;8:1159-72.

2 Kales A, Bixler EO, Cadieux RJ, Schneck DW, Shaw LC, Locke TW, et al. Sleep apnoea in a hypertensive population. Lancet 1984;ii:1005-8.

3 Fletcher EC, DeBehnke RD, Lovoi MS, Gorin AB. Undiagnosed sleep apnea in patients with essential hypertension. Ann Intern Med 1985;103:190-5.

4 Lavie P, Ben-Joseph R, Rubin AE. Prevalence of sleep apnea syndrome among patients with essential hypertension. Am Heart f 1984;108:373-6.

5 Williams AJ, Houston D, Finberg S, Lam C, Kinney JL, Santiago S. Sleep apnea syndrome and essential hypertension. Am $\mathcal{F}$ Cardiol 1985;55:1019-22.

6 Warley ARH, Mitchell JH, Stradling JR. Prevalence of nocturnal hypoxaemia amongst men with mild to moderate hypertension. $Q \mathcal{F ~ M e d ~ 1 9 8 8 ; 6 8 : 6 3 7 - 4 4 .}$

7 Stradling JR, Crosby JH. Relation between systemic hypertension and sleep hypoxaemia or snoring: analysis in 748 men drawn from general practice. $B M \mathcal{F}$ in $7480 ; 300: 75-8$.

8 Douglas NJ, Thomas S, Jan MA. Clinical value of polysomnography. Lancet 1992;339:347-50.

9 Cooper BG, Veale D, Griffiths CJ, Gibson GJ. Value of nocturnal oxygen saturation as a screening test for sleep apnoea. Thorax 1991;46:586-8.

10 Cartwright RD. Effect of sleep position on sleep apnoea severity. Sleep 1984;7:110-4.

11 Hirshkowitz M, Karacan I, Gurakar A, Williams RL. Hypertension, erectile dysfunction, and occult sleep apnea. Sleep 1989;12:223-32.

12 Koskenvuo M, Kaprio J, Partinen M, Langinvainio $H_{\text {, }}$ Sarna S, Heikkla K. Snoring as a risk factor for hypertension and angina pectoris. Lancet 1985;i:893-6.

13 Norton PG, Dunn EV. Snoring as a risk factor for disease: an epidemiological survey. 1985;291:630-2.

14 Mateika JH, Mateika S, Slutsky AS, Hoffstein V. The effect of snoring on mean arterial blood pressure during non-REM sleep. Am Rev Respir Dis 1992;145:141-6.

15 Stradling JR, Crosby JH, Payne CD. Self-reported snoring and daytime sleepiness in men aged 35-65 years. Thorax 1991;46:807-10.

16 Waller PC, Bhopal RS. Is snoring a cause of vascular disease? An epidemiological review. Lancet 1989;i:143-6. 